\title{
The impact of voluntary exercise on relative telomere length in a rat model of developmental stress
}

\author{
Martmari Botha', Laurian Grace ${ }^{2}$, Kishor Bugarith², Vivienne A Russell ${ }^{2}$, Martin Kidd ${ }^{3}$, Soraya Seedat ${ }^{4}$ \\ and Sian MJ Hemmings ${ }^{4^{*}}$
}

\begin{abstract}
Background: Exposure to early adverse events can result in the development of later psychopathology, and is often associated with cognitive impairment. This may be due to accelerated cell aging, which can be catalogued by attritioned telomeres. Exercise enhances neurogenesis and has been proposed to buffer the effect of psychological stress on telomere length. This study aimed to investigate the impact of early developmental stress and voluntary exercise on telomere length in the ventral hippocampus (VH) and prefrontal cortex (PFC) of the rat. Forty-five male Sprague-Dawley rats were categorised into four groups: maternally separated runners (MSR), maternally separated non-runners (MSnR), non-maternally separated runners (nMSR) and non-maternally separated non-runners (nMSnR). Behavioural analyses were conducted to assess anxiety-like behaviour and memory performance in the rats, after which relative telomere length was measured using qPCR.
\end{abstract}

Results: Maternally separated (MS) rats exhibited no significant differences in either anxiety levels or memory performance on the elevated-plus maze and the open field compared to non-maternally separated rats at 49 days of age. Exercised rats displayed increased levels of anxiety on the day that they were removed from the cages with attached running wheels, as well as improved spatial learning and temporal recognition memory compared to non-exercised rats. Exploratory post-hoc analyses revealed that maternally separated non-exercised rats exhibited significantly longer telomere length in the VH compared to those who were not maternally separated; however, exercise appeared to cancel this effect since there was no difference in $\mathrm{VH}$ telomere length between maternally separated and non-maternally separated runners.

Conclusions: The increased telomere length in the VH of maternally separated non-exercised rats may be indicative of reduced cellular proliferation, which could, in turn, indicate hippocampal dysfunction. This effect on telomere length was not observed in exercised rats, indicating that voluntary exercise may buffer against the progressive changes in telomere length caused by alterations in maternal care early in life. In future, larger sample sizes will be needed to validate results obtained in the present study and obtain a more accurate representation of the effect that psychological stress and voluntary exercise have on telomere length.

Keywords: Stress, Exercise, Relative telomere length

\footnotetext{
* Correspondence: smjh@sun.ac.za

${ }^{4}$ Department of Psychiatry, Faculty of Medicine and Health Sciences,

Stellenbosch University, Tygerberg, South Africa

Full list of author information is available at the end of the article
} 


\section{Background}

The development of human physiology and behaviour is a gradual process, which is constantly influenced by the intricate interplay between genetic and environmental factors. In terms of neurodevelopment, the neonatal period is marked by continuous proliferation and maturation of synapses and neurotransmitter pathways [1], rendering the brain sensitive to internal and external stimuli throughout this time. Exposure to adverse experiences during early childhood may therefore have a long-lasting, debilitating impact on several aspects of physiological and psychological development [2,3], ultimately unfolding in anxiety disorders including posttraumatic stress disorder (PTSD).

Although the exact mechanisms through which stress influences physical and psychological wellbeing are presently unclear, increasing evidence supports the involvement of accelerated cell aging in this process [4]. The extent of this progressive aging in eukaryotic organisms can be catalogued by means of telomeres, the guaninerich sections of recurring DNA sequences at the extremities of chromosomes [4-6]. During mitotic cell division, the 3' ends of linear chromosomes are not fully replicated by DNA polymerase [7], resulting in their progressive shortening through the course of each replication cycle [8]. Telomeres play an integral role in maintaining genomic stability and preventing chromosomal end-fusion [9] by enabling replication without the loss of nucleotide bases, and thus genetic information, at the ends of DNA strands. In stem cells and adult germ cells, the erosion of telomeric regions is normally compensated for through the action of telomerase, which inserts essential telomeric nucleotides onto the ends of these structures [10]. However, telomerase activity is virtually undetectable in human and mouse somatic cells [11,12], and for the most part, telomeres in these cells remain shortened. In the event of significant telomere attrition, cell proliferation ceases, leading to a state of senescence or apoptosis [13]. Telomere length (TL) can therefore serve as an indicator of a cell's biological age [4] and predict its future proliferative potential [6].

To a large extent, TL is governed by genetic factors [9], with heritability estimates obtained from family and twin studies ranging between $78 \%$ and $82 \%[14,15]$. In addition, a number of lifestyle factors, such as smoking, sleep duration, body mass index (BMI) and physical activity can alter telomere length $[16,17]$. Shorter TL has been associated with an overall detrimental effect on cardiovascular health [16,18-20] cancer and a spectrum of age-related disorders [21]. In addition, self-perceived psychological stress, as well as the chronicity of stress has been linked to shorter TL in humans $[4,6]$. Similarly, shorter blood leukocyte TL has been reported in caregivers who experience high levels of stress [22] and in patients with mood disorders [23].
Exposure to adverse experiences such as psychological stress may have the greatest neurobiological impact during early development, when the rate of telomere shortening is at its peak and factors involved in TL regulation may be encoded $[24,25]$. Childhood physical and emotional maltreatment has been associated with increased blood leukocyte telomere attrition in psychiatrically healthy adults [26], as well as in individuals with anxiety disorders compared to control individuals [27]. Likewise, shorter leukocyte TL was observed in female mice that were subjected to reproductive stress and their male counterparts exposed to crowding stress, compared to control groups [5].

Not all individuals who experience stress develop chronic pathology, and likewise, not all of these individuals will display shortened DNA telomeric regions. This observation highlights the possibility of supplementary mechanisms that may remediate the effects of stress on telomere shortening. One such approach is physical exercise, which has been linked to longer leukocyte TL, in both trained athletes and a general sample of twins $[17,28]$ Puterman et al. [29] found shorter blood leukocyte TL to be associated with higher levels of perceived stress in non-exercised individuals, whereas this effect seemed to be ameliorated in exercised individuals, thereby supporting the hypothesis that physical exercise may serve as a buffer for the detrimental effects of perceived psychological stress.

TL may serve a role as a biomarker of subsequent stress responses in stress-exposed individuals. Since previous studies have mainly measured TL in peripheral blood mononuclear cells, we sought to investigate the impact of early developmental stress and voluntary exercise on relative telomere length (RTL) in tissues relevant to this process, namely the rat ventral hippocampus (VH) and prefrontal cortex (PFC).

\section{Results}

\section{Behavioural analyses}

The results of the behavioural studies have been reported previously [30]. There were no significant differences in anxiety levels, as measured in the open field (OF) and elevated plus maze (EPM), in maternally separated (MS) and non-maternally separated (nMS) rats at postnatal day 49 (PND49). In contrast, exercised rats (runners, R) exhibited increased levels of anxiety-like behaviour in both of these tests on the day that they were removed from their cages with attached running wheels. Similarly, maternal separation did not produce any change in memory performance. Exercised rats (MSR and nMSR) performed significantly better in the Morris water maze (MWM) and the temporal order task in comparison to non-exercised rats (MSnR and nMSnR). 


\section{Relative telomere length (RTL)}

Slopes of the standard curves plotted from the mean $\mathrm{C}_{\mathrm{q}}$ versus the $\log$ of serial dilution concentrations ranged from -2.26 to -3.31 , yielding corresponding PCR efficiencies between $100 \%$ and $130 \%$ (Table 1). The correlation coefficients for all assays were greater than the generally accepted 0.985. Six of the 44 samples from the $\mathrm{VH}$ assays and 9 of the 43 PFC samples were excluded from further analysis due to non-amplification, $\mathrm{C}_{\mathrm{q}}$ values exceeding 30 or differences in $\mathrm{C}_{\mathrm{q}}$ values between two or more of the triplicates being greater than 0.5 .

Some deviations from the analysis of variance (ANOVA) assumptions were detected; however, further analysis done on log-transformed data yielded essentially the same results. Results for the untransformed data are thus reported. No significant variance was detected in the PFC tissues. In the $\mathrm{VH}$, the two-way ANOVA did not reveal significant effects of stress or exercise $\left(\mathrm{F}_{1,32}=2.24\right.$, $\mathrm{p}=0.14$ ) (Figure 1 and Table 2). However, although the interaction was not significant, post-hoc Fisher's least significant difference (LSD) test revealed that the RTL was significantly longer in the MSnR group in comparison to the $n M S n R$ group $(\mathrm{p}=0.037)$. A non-parametric bootstrap test with Bonferroni post-hoc correction confirmed the trends reported. RTL did not differ significantly when MSR were compared to nMSR, when MSR were compared to MSnR or when nMSR were compared to nMSnR.

Two-way ANOVA revealed that there was no significant effect of stress or voluntary exercise on the RTL in the PFC. Furthermore, the interaction was not significant, and RTL in the PFC did not differ significantly between any of the experimental groups.

\section{Discussion}

Maternal separation paradigms have been used extensively to investigate the impact of early-life adversity, and have been shown to have numerous long-term consequences, including increased levels of anxiety-like behaviour and neurochemical changes, pointing towards HPA axis dysregulation and impaired memory formation [31-33]. However, the maternally separated rats used in the present study did not display increased anxiety-like

Table 1 PCR efficiencies and correlation coefficients for Tel $_{1}$ and $A T_{1}$ assays of the left VH and left PFC

\begin{tabular}{ccccc}
\hline Brain region & Assay & Standard curve slope & PCR efficiency & $\mathbf{R}^{\mathbf{2}}$ \\
\hline VH & $\mathrm{Tel}_{1}$ & -2.86 & $123 \%$ & 0.998 \\
& $\mathrm{AT}_{1}$ & -3.32 & $100 \%$ & 0.986 \\
\multirow{2}{*}{ PFC } & $\mathrm{Tel}_{1}$ & -2.76 & $130 \%$ & 0.994 \\
& $\mathrm{AT}_{1}$ & -3.30 & $100 \%$ & 0.995 \\
\hline
\end{tabular}

$\mathrm{VH}$, ventral hippocampus; PFC, prefrontal cortex; $\mathrm{R}^{2}$, correlation coefficient. behaviour or memory impairment [30]. In terms of anxiety, several authors have failed to detect significant effects of repeated maternal separation [31-36]. This may be a consequence of upregulation of maternal care and increased arched-back nursing, licking and grooming of pups by the dam when they are returned to the cage, which may counteract the stressful effects of maternal separation [37,38]. The inconsistent results may also be due to the use of highly variable maternal separation paradigms between studies [39]. In addition, it is possible that anxiety-like behaviour is not readily observed in adolescence (PND49). To this end, in a separate study, rats subjected to the same maternal separation protocol exhibited anxiety-like behaviour in adulthood (PND75) [40].

Exercised rats used in the present study displayed significantly increased levels of anxiety compared to sedentary controls [30]. This was, however, attributed to the stress of being removed from the running wheels on the same day that the OF and EPM tests were performed [30].

Although no effects of stress or exercise on RTL were observed in the present study, an exploratory post-hoc analysis revealed a significant difference in RTL in the VH between rats in the MSnR and nMSnR groups, while the RTLs of the MSR and nMSR were virtually indistinguishable. MSnR rats were found to possess significantly longer telomeres when compared to nMSnR rats. These results are interesting in light of those obtained by Hulshof et al. [41] who found that, although the maternal separation protocol resulted in no overt differences in anxiety-like behaviour between maternally separated and control Wistar rats, maternally separated rats exhibited significantly reduced cell proliferation in the $\mathrm{VH}$, which the authors suggest could interfere with the normal development of the hippocampus. The results from Hulshof et al. [41] corroborated those obtained in previous studies $[42,43]$. Telomeres reduce in length with each cell division; therefore, the lower the proliferative capabilities of the tissue, the longer the telomeres will be. The increase in RTL in MSnR in the present study may thus be indicative of reduced cellular proliferation in the $\mathrm{VH}$ of those rats, which could indicate alterations in normal development of this brain region. Although the functional implications of reduced proliferative ability in the $\mathrm{VH}$ are currently unknown, the results suggest that voluntary exercise could serve as a buffer against the progressive changes in RTL caused by alterations in maternal care early in life. One way in which exercise may buffer changes in RTL is by moderating the overall burden of oxidative stress on the body, and specifically in the $\mathrm{VH}$, through the upregulation of genes that encode powerful antioxidant enzymes [44]. Indeed, regular, moderate exercise has been shown to increase the body's endogenous antioxidant activity and its resistance to oxidation [45]. 


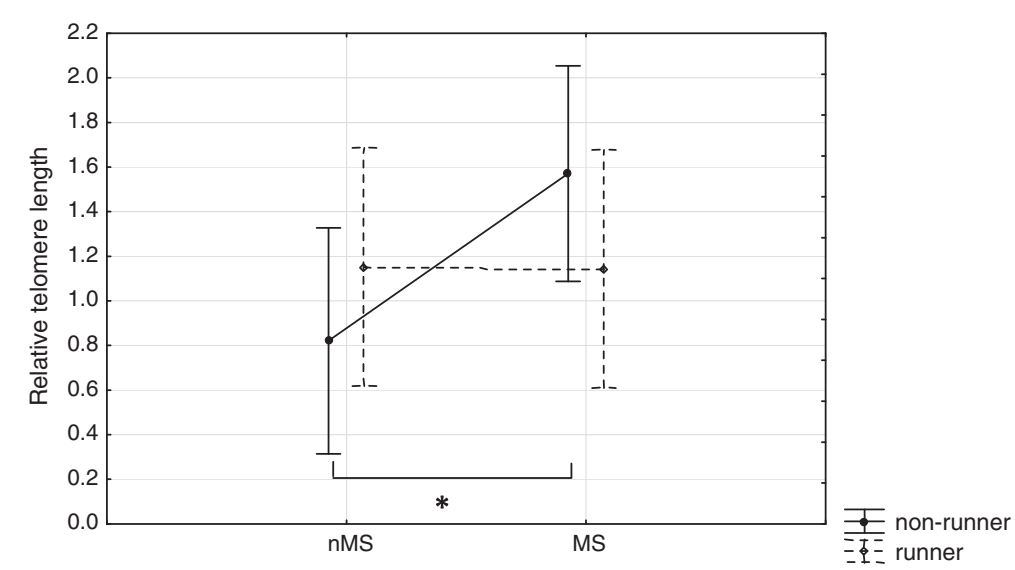

Figure 1 The effect of maternal separation and voluntary exercise on relative telomere length (RTL) in the left ventral hippocampus. ${ }^{*}$ RTL was significantly longer in MSnR compared to nMSnR ( $\left.p=0.037\right)$. Data represent means \pm SEM. Abbreviations: MSR, maternally separated exercised rats; MSnR, maternally separated non-exercised rats; nMSR, non-maternally separated exercised rats; nMSnR, non-maternally separated non-exercised rats.

No further significant differences were detected as a result of maternal separation or exercise in either the $\mathrm{VH}$ or PFC. These results contrast with data from previous studies indicating that psychological stress gives rise to shorter TL in human peripheral blood mononuclear cells and leukocytes $[4-6,26,46]$. However, it is important to note that the vast majority of studies associating shorter TL with stress have been conducted in humans, utilising DNA extracted from peripheral blood mononuclear cells instead of brain tissue. Telomere length has been found to vary in a tissue-specific fashion $[47,48]$. For example, Thomas et al. [49] reported shorter TL in white blood cells and buccal cells, but longer TL in the hippocampi of patients with Alzheimer's disease, compared to control individuals. In addition, results from a recent study indicate that chronic exercise alters TL in a tissue-specific manner [50].

Although multiple factors, including physiology, nutrition, reproductive ability, behaviour and strain should be taken into account, it is generally accepted that most

Table 2 Descriptive data pertaining to the effect of maternal separation and voluntary exercise on RTL in the left ventral hippocampus

\begin{tabular}{lllll}
\hline Experimental group & $\mathbf{n}$ & Mean RTL & SEM & $\mathbf{9 5 \%} \mathbf{C l}$ \\
\hline nMSnR & 10 & 0.821 & 0.061 & $0.682-0.960$ \\
nMSR & 9 & 1.152 & 0.101 & $0.919-1.385$ \\
MSnR & 11 & 1.571 & 0.397 & $0.687-2.454$ \\
MSR & 9 & 1.142 & 0.219 & $0.638-1.647$
\end{tabular}

Abbreviations: nMSnR, non-maternally separated non-runners; nMSR, nonmaternally separated runners; MSnR, maternally separated non-runners; MSR, maternally separated runners; RTL, relative telomere length; SEM, standard error of means; $\mathrm{Cl}$, confidence interval. rats reach maturity at 40 to 60 days of age [51]. It has been proposed that differences in TL might be agerelated, with significant variation only presenting in older cohorts [6]. Since the normal lifespan of rats ranges from two-and-a-half to three years, more distinct differences in RTL might become apparent at a later age. Given that altered TL has been previously associated with both the severity and chronicity of psychological stress [4], as well as the number of adverse early life events in individuals suffering from anxiety disorders [46], stress is likely to have additive and multiplicative effects on RTL.

\section{Conclusions}

The significant results in the present study, although exploratory, are interesting and as such, warrant further investigation in a larger sample. Future studies will benefit from measurements of telomerase activity and markers of oxidative stress, in addition to measurements of RTL in a larger sample group, in order to obtain a more accurate representation of the effect that developmental stress and voluntary exercise might have on RTL.

\section{Methods}

\section{Ethical approval}

All animal experiments were approved by the University of Cape Town's Faculty of Health Sciences Research Ethics Committee. Animal experiments were conducted in accordance with the South African National Guidelines, namely 'The South African National Standard: the care and use of animals for scientific purposes'. The rats were anaesthetised by exposure to halothane vapour 
(Lakato (Pty) Ltd, Cape Town, South Africa) immediately prior to decapitation.

\section{Behavioural analyses}

Forty-five male Sprague-Dawley (Rattus norvegicus) rat pups were housed in culled groups of eight, together with their dams, under standard conditions [30]. Food and water was available ad libitum. Maternal separation was performed as previously described. Briefly, on PND2, rat pups were randomly assigned to one of four experimental groups as follows: maternally separated runners (MSR; $\mathrm{n}=10$ ), maternally separated nonrunners (MSnR; $\mathrm{n}=11)$, non-maternally separated runners (nMSR; $\mathrm{n}=11$ ) and non-maternally separated non-runners (nMSnR; $n=13$ ). On PND2, the dams of pups in the maternally separated group were removed from their home cages and the pups were transferred, in their home cages, to another room in order to prevent communication by ultrasound vocalizations. After 3 hours, the dams were reunited with their pups in the home cage. This protocol was repeated once a day between 9:00 and 13:00 until PND14, after which normal interaction between the dam and her pups resumed.

Pups were weaned on PND21, and voluntary exercise was initiated on PND29 by placing rats (MSR and nMSR) in individual cages with running wheels attached. Non-exercised rats (MSnR and nMSnR) were housed singly in standard plastic cages from PND29 to PND49. Exercised rats were removed from the cages with attached running wheels on PND49 and housed in standard cages in groups of twos or threes until termination of the experiment.

Anxiety-like behaviour was appraised in the $\mathrm{OF}$ and EPM at PND49, as described by Grace et al. [30]. The MWM and several object recognition tasks were used to assess memory performance from PND50 to PND64, as previously described [30].

\section{DNA isolation and preparation}

Rats were decapitated on PND65, after which the VH and PFC were dissected from the left hemisphere of each rat and stored at $-80^{\circ} \mathrm{C}$ in RNAlater ${ }^{\circledR}$ solution. Genomic DNA was purified from these regions using an AllPrep DNA/RNA Mini Kit (Qiagen, Hilden, Germany), with slight modifications made to the standard protocol. The concentration and quality of the DNA were verified by means of the Nanodrop ${ }^{\circledR}$ ND-1000 spectrophotometer (Nanodrop Technologies, Wilmington, DE, USA). Separate calibrator samples consisting of pooled DNA from $44 \mathrm{VH}$ and $43 \mathrm{PFC}$ samples respectively were created and serially diluted to yield eight standards with concentrations ranging from $50 \mathrm{ng} / \mathrm{ul}$ to $1.32 \mathrm{ng} / \mathrm{ul}$.

\section{Quantitative real-time PCR}

Amplification of telomeric DNA (T), together with that of the single-copy genomic $A T_{1}$ receptor (S) was performed on a MicroAmp Optical 384-well Reaction Plate (Applied Biosystems, Foster City, CA, USA) using Applied Biosystem's 7900HT Fast Real-Time polymerase chain reaction (qPCR) system and the slightly modified protocol of Cawthon et al., 2002 [52]. Plates were prepared with the epMotion 5070 (Eppendorf, Hamburg, Germany) so that each $10 \mu \mathrm{l}$ reaction mixture for the telomere reaction $\left(\mathrm{Tel}_{1}\right)$ for both $\mathrm{VH}$ and PFC samples consisted of $5 \mu \mathrm{l}$ QuantiFast $2 \times$ SYBR Green PCR mastermix (Qiagen, Hilden, Germany), $270 \mathrm{nM}$ forward primer (5'-GGTTTTTGAGGGTGAGGGTGAGGGTGAGGGTGA GGGT-3'), $900 \mathrm{nM}$ reverse primer (5'-TCCCGACTAT CССТАТСССТАТСССТАТСССТАТСССТА-3'), $25 \mathrm{ng}$ genomic DNA and water. The specific reaction mix for $A T_{1}$ was similar except that it included $800 \mathrm{nM}$ forward primer (5'-ACGTGTTCTCAGCATCGACCGCTACC-3') and $600 \mathrm{nM}$ reverse primer (5'-AGAATGATAAGG $\left.A A A G G G A A C A A G A A G C C C-3^{\prime}\right)$ for the $\mathrm{VH}$ samples, and $700 \mathrm{nM}$ and $500 \mathrm{nM}$ of forward and reverse primer respectively for the PFC samples. All samples were run in triplicate in order to account for possible technical variation. Cycling conditions were as follows: $95^{\circ} \mathrm{C}$ for 5 minutes, followed by 40 cycles of $95^{\circ} \mathrm{C}$ for 10 seconds and $54^{\circ} \mathrm{C}$ for 30 seconds in the case of the $\mathrm{VH}$ and PFC $\mathrm{Tel}_{1}$ and PFC $A T_{1}$ assays, or $60^{\circ} \mathrm{C}$ in the case of the $\mathrm{VH}$ $A T_{1}$ assay.

Rigorous control measures were put in place to ensure the quality of the data. An additional melting curve analysis consisting of $95^{\circ} \mathrm{C}$ for 15 seconds, $60^{\circ} \mathrm{C}$ for 15 seconds and $95^{\circ} \mathrm{C}$ for 15 seconds was performed at the end of each reaction to verify specific amplification. The serial dilution series was used in standard curve construction, and all quantification cycle $\left(C_{\mathrm{q}}\right)$ values were corrected according to efficiencies calculated from the standard curve. Threshold and baseline values were automatically determined by the Sequence Detector Systems (SDS) version 2.4 software. Samples with a difference in $C_{q}$ values greater than 0.5 between triplicates were disregarded in further analyses.

\section{Data analysis}

The RTL was determined as the factor by which the experimental sample differed from the reference sample in its ratio of telomere repeat copy number $(\mathrm{T})$ to the $A T_{1}$ copy number (S) [52]. A two-way ANOVA was applied to the data, followed by post-hoc Fisher's LSD tests. A non-parametric bootstrap test with Bonferroni post-hoc correction was performed to eliminate the possibility of unequal variances. Statistically significant results with $\mathrm{p}$ values of less than 0.05 are reported. 


\section{Abbreviations}

TL: Telomere length; RTL: Relative telomere length; VH: Ventral hippocampus; PFC: Prefrontal cortex; PND: Postnatal day; MSR: Maternally separated runners; MSnR: Maternally separated non-runners; nMSR: Non-maternally separated runners; nMSnR: Non-maternally separated non-runners; OF: Open field; EPM: Elevated-plus maze; MWM: Morris water maze.

\section{Competing interest}

The authors declare that they have no competing interests.

\section{Authors' contributions}

MB carried out the molecular genetic studies and drafted the initial manuscript. LG carried out the behavioural analyses and assisted with drafting the manuscript. KB carried out behavioural analyses and participated in the design of the study. VR conceived of the behavioural study and assisted in drafting the manuscript. MK performed the statistical analyses. SS participated in the design and coordination of the study and assisted with drafting the manuscript. SH conceived of the idea, participated in coordination of the design of the study and assisted in drafting the manuscript. All authors read and approved the final manuscript.

\section{Acknowledgements}

This work is based upon research supported by the South African Research Chairs Initiative of the Department of Science and Technology (DST), the National Research Foundation (NRF) and the Harry Crossley Foundation The authors would also like to acknowledge the Medical Research Council (MRC) Centre for Molecular and Cellular Biology, Tygerberg, South Africa.

\section{Author details}

${ }^{1}$ Department of Biomedical Sciences, Faculty of Medicine and Health Sciences, Stellenbosch University, Tygerberg, South Africa. ${ }^{2}$ Department of Human Biology, University of Cape Town, Cape Town, South Africa. ${ }^{3}$ Centre for Statistical Consultation, Stellenbosch University, Stellenbosch, South Africa. ${ }^{4}$ Department of Psychiatry, Faculty of Medicine and Health Sciences, Stellenbosch University, Tygerberg, South Africa.

Received: 24 August 2012 Accepted: 18 December 2012

Published: 27 December 2012

\section{References}

1. Clancy B, Darlington RB, Finlay BL: Translating developmental time across mammalian species. Neuroscience 2001, 105:7-17.

2. Gareau MG, Jury J, Yang PC, MacQueen G, Perdue MH: Neonatal maternal separation causes colonic dysfunction in rat pups including impaired host resistance. Pediatr Res 2006, 59:83-88.

3. Horvath KM, Harkany T, Mulder J, Koolhaas JM, Luiten PG, Meerlo P: Neonatal handling increases sensitivity to acute neurodegeneration in adult rats. J Neurobiol 2004, 60:463-472.

4. Epel ES, Blackburn EH, Lin J, Dhabhar FS, Adler NE, Morrow JD, et al: Accelerated telomere shortening in response to life stress. Proc Natl Acad Sci USA 2004, 101:17312-17315.

5. Kotrschal A, Ilmonen P, Penn DJ: Stress impacts telomere dynamics. Biol Lett 2007, 3:128-130

6. Parks CG, Miller DB, McCanlies EC, Cawthon RM, Andrew ME, DeRoo LA, et al: Telomere length, current perceived stress, and urinary stress hormones in women. Cancer Epidemiol Biomarkers Prev 2009, 18:551-560.

7. Chan SR, Blackburn EH: Telomeres and telomerase. Philos Trans R Soc Lond B Biol Sci 2004, 359:109-121.

8. Callen $\mathrm{E}$, Surralles J: Telomere dysfunction in genome instability syndromes. Mutat Res 2004, 567:85-104.

9. Bischoff C, Graakjaer J, Petersen HC, Hjelmborg JB, Vaupel JW, Bohr V, et al: The heritability of telomere length among the elderly and oldest-old. Twin Res Hum Genet 2005, 8:433-439.

10. Blackburn $\mathrm{EH}$ : The telomere and telomerase: nucleic acid-protein complexes acting in a telomere homeostasis system. A review. Biochemistry (Mosc) 1997, 62:1196-1201.

11. Counter CM, Avilion AA, LeFeuvre CE, Stewart NG, Greider CW, Harley CB, et al: Telomere shortening associated with chromosome instability is arrested in immortal cells which express telomerase activity. EMBO J 1992, 11:1921-1929.
12. Kipling $D$ : Telomere structure and telomerase expression during mouse development and tumorigenesis. Eur J Cancer 1997, 33:792-800.

13. Olovnikov AM: A theory of marginotomy. The incomplete copying of template margin in enzymic synthesis of polynucleotides and biological significance of the phenomenon. J Theor Biol 1973, 41:181-190.

14. Vasa-Nicotera $M$, Brouilette $S$, Mangino $M$, Thompson JR, Braund $P$, Clemitson JR, et al: Mapping of a major locus that determines telomere length in humans. Am J Hum Genet 2005, 76:147-151.

15. Jeanclos E, Schork NJ, Kyvik KO, Kimura M, Skurnick JH, Aviv A: Telomere length inversely correlates with pulse pressure and is highly familial. Hypertension 2000, 36:195-200.

16. Valdes AM, Andrew T, Gardner JP, Kimura M, Oelsner E, Cherkas LF, et al: Obesity, cigarette smoking, and telomere length in women. Lancet 2005, 366:662-664.

17. Cherkas LF, Hunkin JL, Kato BS, Richards JB, Gardner JP, Surdulescu GL, et al: The association between physical activity in leisure time and leukocyte telomere length. Arch Intern Med 2008, 168:154-158.

18. Gardner JP, Li S, Srinivasan SR, Chen W, Kimura M, Lu X, et al: Rise in insulin resistance is associated with escalated telomere attrition. Circulation 2005, 111:2171-2177.

19. Aviv A, Aviv H: Telomeres and essential hypertension. Am J Hypertens 1999, 12:427-432.

20. Cawthon RM, Smith KR, O'Brien E, Sivatchenko A, Kerber RA: Association between telomere length in blood and mortality in people aged 60 years or older. Lancet 2003, 361:393-395.

21. Blackburn EH: Switching and signaling at the telomere. Cell 2001, 106:661-673.

22. Damjanovic AK, Yang Y, Glaser R, Kiecolt-Glaser JK, Nguyen H, Laskowski B, et al: Accelerated telomere erosion is associated with a declining immune function of caregivers of Alzheimer's disease patients. $J$ Immunol 2007, 179:4249-4254.

23. Simon NM, Smoller JW, McNamara KL, Maser RS, Zalta AK, Pollack MH, et al: Telomere shortening and mood disorders: preliminary support for a chronic stress model of accelerated aging. Biol Psychiatry 2006, 60:432-435

24. Zeichner SL, Palumbo P, Feng Y, Xiao X, Gee D, Sleasman J, et al: Rapid telomere shortening in children. Blood 1999, 93:2824-2830.

25. Cameron N, Demerath EW: Critical periods in human growth and their relationship to diseases of aging. Am J Phys Anthropol 2002, 35(Suppl):159-184.

26. Tyrka AR, Price LH, Kao HT, Porton B, Marsella SA, Carpenter LL: Childhood maltreatment and telomere shortening: preliminary support for an effect of early stress on cellular aging. Biol Psychiatry 2010, 67:531-534.

27. O'Donovan A, Epel E, Lin J, Wolkowitz O, Cohen B, Maguen S, et al: Childhood trauma associated with short leukocyte telomere length in posttraumatic stress disorder. Biol Psychiatry 2011, 70:465-471.

28. Werner C, Furster T, Widmann T, Poss J, Roggia C, Hanhoun M, et al: Physical exercise prevents cellular senescence in circulating leukocytes and in the vessel wall. Circulation 2009, 120:2438-2447.

29. Puterman E, Lin J, Blackburn E, O'Donovan A, Adler N, Epel E: The power of exercise: buffering the effect of chronic stress on telomere length. PLOS One 2010, 5:e10837.

30. Grace L, Hescham S, Kellaway LA, Bugarith K, Russell VA: Effect of exercise on learning and memory in a rat model of developmental stress. Metab Brain Dis 2009, 24:643-657.

31. Biagini G, Pich EM, Carani C, Marrama P, Agnati LF: Postnatal maternal separation during the stress hyporesponsive period enhances the adrenocortical response to novelty in adult rats by affecting feedback regulation in the CA1 hippocampal field. Int J Dev Neurosci 1998 16:187-197.

32. Crnic LC, Bell JM, Mangold R, Gruenthal M, Eiler J, Finger S: Separationinduced early malnutrition: maternal, physiological and behavioral effects. Physiol Behav 1981, 26:695-707.

33. Kalinichev M, Easterling KW, Plotsky PM, Holtzman SG: Long-lasting changes in stress-induced corticosterone response and anxiety-like behaviors as a consequence of neonatal maternal separation in Long-Evans rats. Pharmacol Biochem Behav 2002, 73:131-140.

34. Ogawa T, Mikuni M, Kuroda Y, Muneoka K, Mori KJ, Takahashi K: Periodic maternal deprivation alters stress response in adult offspring: potentiates the negative feedback regulation of restraint stress-induced 
adrenocortical response and reduces the frequencies of open field-induced behaviors. Pharmacol Biochem Behav 1994, 49:961-967.

35. von Hoersten S, Dimitrijevic M, Markovic BM, Jankovic BD: Effect of early experience on behavior and immune response in the rat. Physiol Behav 1993, 54:931-940.

36. Faure J, Uys JD, Marais L, Stein DJ, Daniels WM: Early maternal separation alters the response to traumatization: resulting in increased levels of hippocampal neurotrophic factors. Metab Brain Dis 2007, 22:183-195.

37. Macri S, Chiarotti F, Wurbel H: Maternal separation and maternal care act independently on the development of HPA responses in male rats. Behav Brain Res 2008, 191:227-234.

38. Marmendal M, Eriksson CJ, Fahlke C: Early deprivation increases exploration and locomotion in adult male Wistar offspring. Pharmacol Biochem Behav 2006, 85:535-544.

39. Lehmann J, Feldon J: Long-term biobehavioral effects of maternal separation in the rat: consistent or confusing? Rev Neurosci 2000, 11:383-408.

40. Makena N, Bugarith K, Russell VA: Maternal separation enhances object location memory and prevents exercise-induced MAPK/ERK signalling in adult Sprague-Dawley rats. Metab Brain Dis 2012, 27:377-385.

41. Hulshof HJ, Novati A, Sgoifo A, Luiten PG, den Boer JA, Meerlo P: Maternal separation decreases adult hippocampal cell proliferation and impairs cognitive performance but has little effect on stress sensitivity and anxiety in adult Wistar rats. Behav Brain Res 2011, 216:552-560.

42. Aisa B, Elizalde N, Tordera R, Lasheras B, Del RJ, Ramirez MJ: Effects of neonatal stress on markers of synaptic plasticity in the hippocampus: implications for spatial memory. Hippocampus 2009, 19:1222-1231.

43. Mirescu C, Peters JD, Gould E: Early life experience alters response of adult neurogenesis to stress. Nat Neurosci 2004, 7:841-846.

44. Gomez-Cabrera MC, Domenech E, Vina J: Moderate exercise is an antioxidant: upregulation of antioxidant genes by training. Free Radic Biol Med 2008, 44:126-131.

45. Elosua R, Molina L, Fito M, Arquer A, Sanchez-Quesada JL, Covas MI, et al: Response of oxidative stress biomarkers to a 16-week aerobic physical activity program, and to acute physical activity, in healthy young men and women. Atherosclerosis 2003, 167:327-334.

46. Kananen L, Surakka I, Pirkola S, Suvisaari J, Lonngvist J, Peltonen L, et al: Childhood adversities are associated with shorter telomere length at adult age both in individuals with an anxiety disorder and controls. PLoS One 2010, 5:e10826.

47. Prowse KR, Greider CW: Developmental and tissue-specific regulation of mouse telomerase and telomere length. Proc Natl Acad Sci USA 1995, 92:4818-4822.

48. Wang JC, Warner JK, Erdmann N, Lansdorp PM, Harrington L, Dick JE: Dissociation of telomerase activity and telomere length maintenance in primitive human hematopoietic cells. Proc Natl Acad Sci USA 2005, 102:14398-14403.

49. Thomas P: O' Callaghan NJ, Fenech M: Telomere length in white blood cells, buccal cells and brain tissue and its variation with ageing and Alzheimer's disease. Mech Ageing Dev 2008, 129:183-190.

50. Ludlow AT, Witkowski S, Marshall MR, Wang J, Lima LC, Guth LM, et al: Chronic Exercise Modifies Age-Related Telomere Dynamics in a TissueSpecific Fashion. J Gerontol A Biol Sci Med Sci 2012, doi:10.1093/gerona/ gls002.

51. Kohn D, Clifford C: Biology and diseases of rats. In Laboratory animal medicine. Edited by Fox JG, Anderson LC, Loew FM, Quimby FW. New York: Academic Press.Ref Type: Generic; 2012:121-165.

52. Cawthon RM: Telomere measurement by quantitative PCR. Nucleic Acids Res 2002, 30:e47.

doi:10.1186/1756-0500-5-697

Cite this article as: Botha et al:: The impact of voluntary exercise on relative telomere length in a rat model of developmental stress. BMC Research Notes 2012 5:697.

\section{Submit your next manuscript to BioMed Central and take full advantage of:}

- Convenient online submission

- Thorough peer review

- No space constraints or color figure charges

- Immediate publication on acceptance

- Inclusion in PubMed, CAS, Scopus and Google Scholar

- Research which is freely available for redistribution 